\title{
Hemodynamic effects of cycle ergometer in critical cancer patients: relation length of stay and type of treatment
}

\author{
Wylisson Marcelo Almeida Lins', Ana Cristina Machado Leão', Anke Bergmann'
}

\begin{abstract}
Introduction: In recent years, with the implementation of new chemotherapy protocols, radiation and surgery, there was an improvement in cancer patient's prognosis. However, both the treatment and the disease itself generate a series of complications, worsening clinical condition, which may lead to the need for admission to the intensive care unit (ICU). Physical therapy works on physical and functional recovery of these individuals, using manual and / or mechanical features, among them the cycle ergometer is gaining prominence, but there is in the literature any study evaluating the use of this technique in critical cancer patients. Objective: To evaluate the influence length of stay and type of treatment on hemodynamic behavior in response to an active cycle ergometer in cancer patients in intensive care. Methods: We performed a single active intervention cycle ergometer lower limb (no load) for 10 minutes. The variables included heart rate and mean arterial blood pressure and systolic blood pressure, which were evaluated in four stages: rest, 5 and10 minutes of activity and 10 minutes of recovery. Data on the patient and his or her medical history were previously obtained from their medical records. Results: The study included 23 patients, most were males; under the age of 65; hospital stays shorter than 14 days. It was observed that elderly patients, females, patients with a long hospital stay greater than 14 days, and patients in a clinical hospital showed higher variations in heart rate, mean arterial blood pressure and systolic blood pressure during the entire activity. Conclusion: The results of this study indicate that no individual had clinical complications during exercise. Hemodynamic changes occurred more frequently in the group of elderly patients, clinical and longer hospital stays.
\end{abstract}

Keywords: Physiotherapy; Intensive care units; Hemodynamics; Neoplasms; Exercise; Heart rate.

\section{INTRODUCTION}

In recent years, with the implementation of new chemotherapy protocols, radiation and surgery, there was an improvement in cancer patients' prognosis with increased disease-free survival and overall survival. However, both the treatment and the disease itself generate a number of complications, which imply a physiological depletion framework, which can lead to hospitalization need in the intensive care unit (ICU) due to the worsening of the clinical condition. ${ }^{(1,2)}$

During the hospital stay in ICU, patients may have a decreased activity level due to prolonged bed rest; as a result, there is a functional decline, which often leads to immobilism ${ }^{(3)}$.One of the main systems affected by immobilism is musculoskeletal, since seven days of bed rest can determine reduced muscle strength by $30 \%$, with an additional loss of $20 \%$ of the remaining force every week, by changing the electrical activity of excitatory of muscle fibers and decreased muscle endurance. ${ }^{(4,5)}$

Prolonged rest affects other systems, such as the cardiovascular system, and causes a reduction in cardiac reserve, increased heart rate during submaximal exercise, decreased blood flow in the calf and increased fibrinogen levels in the blood with a greater chance of developing deep vein thrombosis. In the respiratory system, immobilism can reduce the functional reserve capacity and functional capacity as well as increase secretion in the airways. ${ }^{(5)}$

Physical therapy is playing an important role in the physical, functional recovery of these individuals, through early mobilization, favoring respiratory improvement, reducing the adverse effects of immobility, improving the level of awareness and cardiovascular fitness and promoting increased functional independence. ${ }^{(6)}$

The early mobilization protocols, studied and currently applied, are compounds, in general, of the following: passive stretching, passive mobilization, joint position, active-assisted exercise, transfer from lying to sitting, active exercise, cycle ergometer, transfer from sitting to chair, orthostatic posture and resistance exercise. ${ }^{(7,8)}$

Among these, the cycle ergometer has been highlighted in recent studies in which its implementation in the treatment protocol is presented as an important resource in intervention with good acceptance and good adaptation by critically 
ill patients. However, most of these studies assessing the feasibility and effectiveness of early mobilization in clinical and functional recovery of critically ill patients usually does not include cancer patients, probably because they are considered more serious and/or unstable by their disease condition and specific treatment. ${ }^{(9,10)}$

To date, there are no studies in the literature to evaluate the use of this technique in cancer patients. The aim of this study was to evaluate the influence of age, length of stay and type of treatment in the hemodynamic behavior in response to active cycle ergometer in cancer patients in intensive care.

\section{METHODS}

The present study was a case series carried out in the Intensive Care Units and Postoperative Hospital Cancer I of the National Cancer Institute José Alencar Gomes da Silva-INCA from June to October 2015. The Research Ethics Committee (CEP-INCA), protocol 1.046.759, approved the study.

Study participants were cancer patients over the age of 18 , spontaneously breathing, with or without the use of oxygen support, awake, with a score on the Glasgow coma scale greater/equal to $11^{(11)}$, with a degree of muscle strength in the lower limbs (LL) greater/equal to 3 (according to the Manual Muscle Test [MMT]) ${ }^{(12)}$, hemodynamically stable, even in vasoactive amines, and who have agreed to participate in the study by signing a free and informed consent.

Exclusion criteria were defined as the presence of musculoskeletal disorders that impede the mobilization of the lower limbs, deep vein thrombosis of the lower limbs, without treatment or within 48 hours of onset of anticoagulation therapy, tumors in the lower limbs, previous neurological disorders, congestive heart failure grade IV and myocardial infarction shorter than 72 hours, unstable fracture vertebral bodies, platelet count below 10,000 ul and/or hemoglobin below $6 \mathrm{dl}$, active bleeding, and presence of a hemodialysis catheter in the femoral vein or during hemodialysis. (Figure 1) details the steps of selecting the individuals.

Considering a change of $5 \%$ between the follow-up measurements, with an absolute accuracy of $10 \%$ and $5 \%$ significance level was necessary to include at least 18 volunteers. During the study period, 23 volunteers were eligible, and this is the population studied.

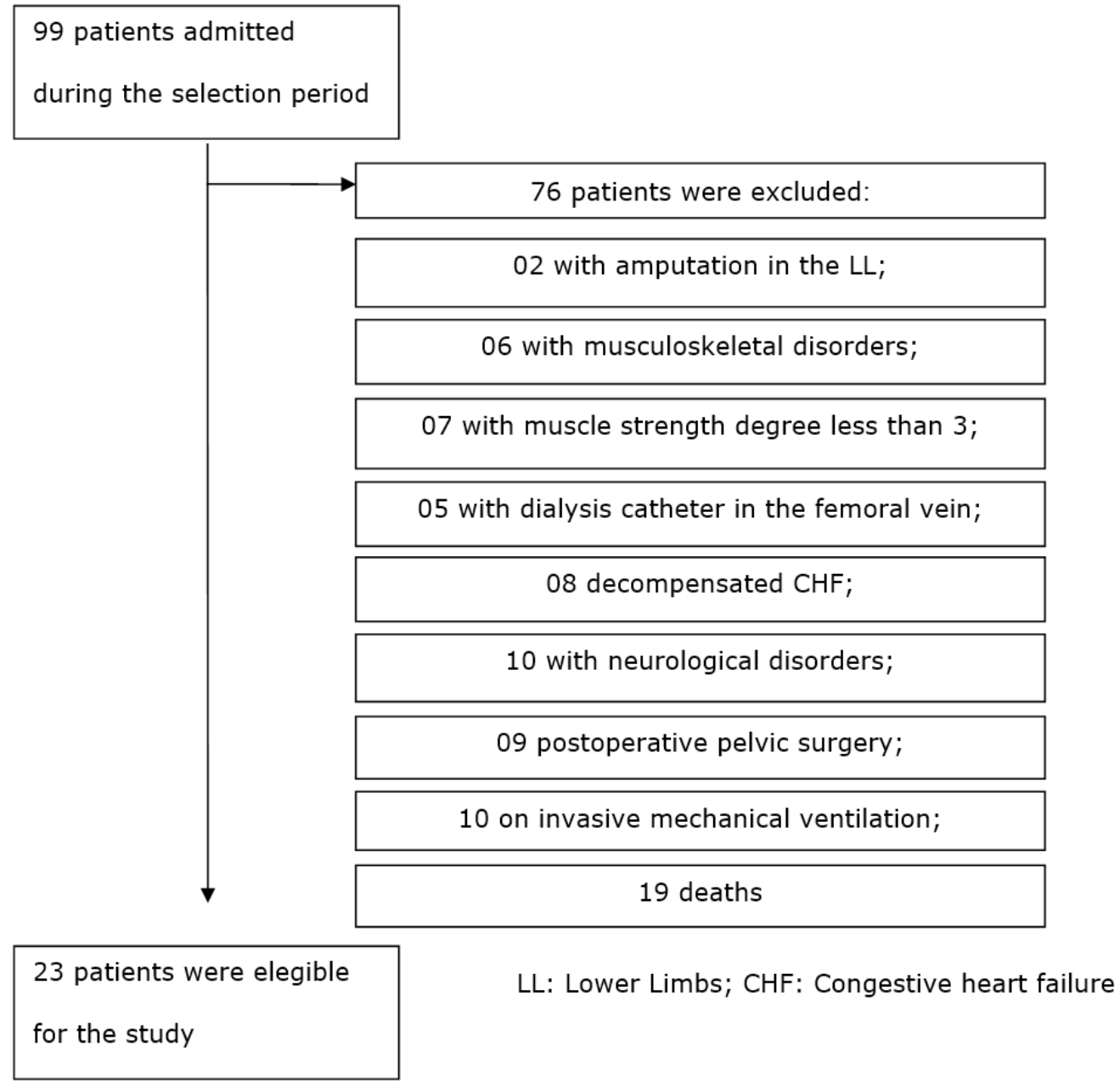

Figure 1. Flowchart of the selection of eligible patients 


\section{Study protocol}

To start the activity, patients were placed in a sitting position at their bedside, with dorsal support. A cycle ergometer ( $\mathrm{ACTE}^{\circledR}$, São Paulo, Brazil) was coupled to the lower limbs and the modified Borg scale was explained, for the control of exercise intensity. When the researcher directed, the patient began to actively pedal a cycle ergometer (no charge). Each patient was instructed to pedal the fastest possible speed, keeping pace throughout the experiment. The activity was 10 minutes duration, and the protocol was performed once during the patient's hospital stay.

The values of heart rate (HR), systolic blood pressure (SBP) and mean arterial pressure (MAP) were measured before the activity, at 5 and 10 minutes of activity and after 10 minutes after exercise (recovery period). The signals were measured by a monitor (Phillips DX2020, Manaus, Brazil).

Data on the patient and his or her medical history were previously obtained from their medical records, such as age ( $<65$ years and $\geq 65$ years), gender, length of stay ( $\leq 14$ days or $>14$ days), cause of hospitalization (clinical or surgical), type of ventilation (room air or oxygen therapy), vasoactive amines, prior treatment (chemotherapy, radiotherapy and surgery). The muscle strength of the lower limbs was measured before the intervention.

In case of hemodynamic instability where the patient had HR: less than 40 bpm or greater than 130 bpm; RR: less than $5 \mathrm{bpm}$ or higher $40 \mathrm{bpm}$; SBP: greater than $180 \mathrm{mmHg}$; MAP: less than $65 \mathrm{mmHg}$ or greater than $110 \mathrm{mmHg}$; SpO2: less than 90\%; Borg: greater than 8 or reporting some kind of discomfort, such as chest pain, shortness of breath, pain in lower limbs, dizziness, exercise was stopped, and the data obtained so far were recorded.

\section{Statistical analysis}

Categorical variables were presented as absolute numbers and proportions and continuous as mean and standard deviation $( \pm S D)$. The normality of the data for continuous outcomes was confirmed by the Kolmogorov-Smirnov test $(p \geq 0.05)$. For comparison between the moments before, during and after the cycle ergometer test, we used the analysis of variance (ANOVA) for repeated measures with the Bonferroni post-test; to evaluate the absolute change in the parameters pre and post intervention, was used the average variance and to evaluate the relative change was used: initial mean- final mean/ initial mean $\times 100$. The data were analyzed using the Statistical Package for Social Sciences (SPSS 17.0), adopting the significance level of $p<0.05$.

\section{RESULTS}

(Table 1) shows the general characteristics of the sample, which included mainly males, under the age of 65 and with hospital stays shorter than 14 days. As the cause for current hospitalization was mostly associated surgical patients.
(Table 2) describes the mean \pm standard deviation of the values obtained in the HR, MAP, SBP measurements, according to the cycle ergometer performace period. An increase of the variables HR, MAP, SBP were observed in the first 5 minutes of activity; in course of the activity, the amounts of PAM, SBP decreased gradually up to 10 minutes of recovery while maintaining lower levels than the rest. In contrast, $\mathrm{HR}$ remained stable during up to 10 minutes of the activity; it decreased during the recovery period but showed a higher value than at rest.

We notice that the changes in the variables occurred mainly at the beginning of the activity when compared to the whole evaluation. When we look at the initial data to 5 minutes of activity; heart rate changed $12.43 \%$, mean blood pressure $6.13 \%$ and systolic blood pressure $9.65 \%$. During the 10 minute interval of activity $x 10$ minute recovery, there was a reduction of $-8.08 \%$ in heart rate, $-8.09 \%$ mean blood pressure, and $-10.59 \%$ the systolic blood pressure (Table 3 ).

The (Table 4) compared the effects of the activity with the independent variables (gender, age, length of stay and

Table 1. Sample characteristics evaluated

\begin{tabular}{|c|c|c|}
\hline Variable & $\mathbf{n}$ & $\%$ \\
\hline \multicolumn{3}{|l|}{ Gender } \\
\hline Male & 13 & 56.5 \\
\hline Female & 10 & 43.5 \\
\hline \multicolumn{3}{|l|}{ Age } \\
\hline$<65$ years & 14 & 60.9 \\
\hline$\geq 65$ years & 9 & 39.1 \\
\hline \multicolumn{3}{|c|}{ Length of hospital stay } \\
\hline$\leq 14$ days & 16 & 69.6 \\
\hline$>14$ days & 7 & 30.4 \\
\hline \multicolumn{3}{|c|}{ Type of hospitalization } \\
\hline Clinical & 10 & 43.5 \\
\hline Surgical & 13 & 56.5 \\
\hline \multicolumn{3}{|l|}{ Respiratory status } \\
\hline Room Air & 14 & 60.9 \\
\hline Oxygen therapy & 9 & 39.1 \\
\hline \multicolumn{3}{|l|}{ Vasoactive Drug } \\
\hline Yes & 1 & 4.3 \\
\hline No & 22 & 95.7 \\
\hline \multicolumn{3}{|l|}{ Early treatment } \\
\hline \multicolumn{3}{|l|}{ Chemotherapy } \\
\hline Yes & 11 & 47.8 \\
\hline No & 12 & 52.2 \\
\hline \multicolumn{3}{|l|}{ Radiotherapy } \\
\hline Yes & 7 & 30.4 \\
\hline No & 16 & 69.6 \\
\hline
\end{tabular}


Table 2. Characteristics of variables during activity

\begin{tabular}{|c|c|c|c|c|}
\hline \multirow{2}{*}{ Variable } & Rest & $5 \mathrm{~min}$. activi. & $10 \mathrm{~min}$. activi. & $10 \mathrm{~min}$. recov. \\
\hline & Mean \pm SD & Mean \pm SD & Mean \pm SD & Mean \pm SD \\
\hline $\mathrm{HR}$ (Beats/min) & $98.0 \pm 24.3$ & $110.3 \pm 13.7$ & $110.5 \pm 14.8$ & $102.4 \pm 16.0$ \\
\hline $\mathrm{MAP}(\mathrm{mmHg})$ & $99.0 \pm 23.2$ & $105.1 \pm 22.0$ & $99.5 \pm 20.5$ & $92.5 \pm 13.5$ \\
\hline $\mathrm{SBP}(\mathrm{mmHg})$ & $134.0 \pm 28.2$ & $143.7 \pm 27.8$ & $139.2 \pm 26.8$ & $128.8 \pm 18.3$ \\
\hline
\end{tabular}

HR: Heart Rate; MAP: Mean Arterial Pressure ; SBP: Systolic Blood Pressure ; mmHg: Millimeters of Mercury; min.: Minutes; activi.: Activity; recov.: Recovery. Results expressed as mean \pm standard deviation.

Table 3. Hemodynamic parameters after an active cycle ergometer according to the time

\begin{tabular}{|c|c|c|}
\hline \multirow{2}{*}{ Hemodynamic parameters } & $\begin{array}{l}\text { Absolute } \\
\text { Alteration }\end{array}$ & $\begin{array}{l}\text { Relative } \\
\text { Alteration }\end{array}$ \\
\hline & Mean \pm SD & (\%) \\
\hline \multicolumn{3}{|l|}{ HR (beats/min) } \\
\hline Rest $\rightarrow 5 \mathrm{~min}$. activi. & $12.43 \pm 22.45$ & 10.96 \\
\hline Rest $\rightarrow 10$ min. activi. & $12.60 \pm 23.09$ & 10.89 \\
\hline $\begin{array}{l}10 \mathrm{~min} . \text { activi. } \rightarrow 10 \mathrm{~min} \text {. } \\
\text { recov. }\end{array}$ & $-8.08 \pm 7.01$ & -8.09 \\
\hline \multicolumn{3}{|l|}{ MAP (mmHg) } \\
\hline Rest $\rightarrow 5$ min. activi. & $6.13 \pm 26.82$ & 3.74 \\
\hline Rest $\rightarrow 10$ min. activi. & $0.52 \pm 25.29$ & -2.20 \\
\hline $\begin{array}{l}10 \mathrm{~min} . \text { activi. } \rightarrow 10 \mathrm{~min} . \\
\text { recov. }\end{array}$ & $-7.04 \pm 11.15$ & -7.14 \\
\hline \multicolumn{3}{|l|}{$\mathrm{SBP}(\mathrm{mmHg})$} \\
\hline Rest $\rightarrow 5$ min. activi. & $9.65 \pm 21.09$ & 5.97 \\
\hline Rest $\rightarrow 10$ min. activi. & $5.17 \pm 17.42$ & 3.26 \\
\hline $\begin{array}{l}10 \mathrm{~min} \text {. activi. } \rightarrow 10 \mathrm{~min} \text {. } \\
\text { recov. }\end{array}$ & $-10.59 \pm 16.79$ & -7.90 \\
\hline
\end{tabular}

HR: Heart Rate; MAP: Mean Arterial Pressure; SBP: Systolic Blood Pressure; mmHg: Millimeters of Mercury; min.: Minutes; activi.:Activity; recov.: Recovery; SD:Standard Deviation. Results obtained by average variance and to evaluate the relative change was used: initial mean- final mean / initial mean x 100 .

Type of hospitalization). Although not statistically significant, individuals with a length of stay greater than 14 days and a stay in a clinical hospital showed higher variation in heart rate, mean arterial pressure, and systolic blood pressure throughout the activity when compared to individuals with hospital stays shorter than 14 days and surgical hospitalization. Likewise, in female subjects and patients older than 65 years, the heart rate and mean arterial pressure ranged throughout the activity, compared to males and younger than 65; on the other hand, when evaluating the systolic blood pressure, males and patients younger than 65 years had a higher variation compared to females and patients older than 65 years.

\section{DISCUSSION}

In our study, the use of a cycle ergometer promoted as a response in the opening minutes an increase in $H R, S B P, M A P$, demonstrating positive hemodynamic effects. After 10 minutes of activity, the variables MAP and SBP decreased compared to 10 minutes of activity, signaling a return to baseline.

While performing the physical activity, mechanical stress causes an increase in blood flow to the muscles in action, since it is necessary to increase the supply of oxygen and nutrients and remove metabolic slag, such as carbon dioxide, lactate and hydrogen ions. Thus, the cardiovascular system is influenced by neural and metabolic stimuli to increase activity. ${ }^{(13)}$

Concerning to heart rate, the physiological mechanism related to hemodynamic responses during an aerobic exercise occurs by decreased parasympathetic tone and increased sympathetic tone, triggered by the motor cortex and afferent neurons (mechanoreceptors and chemoreceptors), which transmit the information to the cardiovascular center increasing cardiac sympathetic tone by releasing a greater amount of noradrenaline, triggering higher activity of the sinoatrial node, and consequently, increasing heart rate. ${ }^{(13,14)}$

Heart rate must be carefully monitored during mobilization: it provides data to assess the patient's exercise tolerance. However, it should not be evaluated in isolation, since when there is an increase in HR during the activity, the patient may experience some signs and symptoms of heart stress such as breathlessness, chest pain, weakness and excessive secretion in the airways ${ }^{(15)}$. Thus, the intensity should be decreased during mobilization and if necessary, the activity should be suspended. In our study, no individual showed exercise intolerance.

In the present study, there was an increase of HR during the 5 and 10 minute activity, with a mean increase of $10.96 \%$ and $10.89 \%$, respectively, for resting $\mathrm{HR}$, as well as a reduction average of $-8.09 \%$ in the range 10 minutes activity $\times 10$ minutes recovery. Similar data can be observed in some studies involving patients in ICUs, where the subjects performed both active and passive mobilization and both resulted in a significant increase in $\mathrm{HR}$ relative to baseline, with the magnitude of the increase of approximately $10 \%{ }^{(16,17)}$. In another study in which the cycle ergometer was applied, an increase of $3 \%$ in heart rate from baseline was identified. ${ }^{(9)}$

It was shown that in the mobilization, even if passive, large muscle groups, especially in the lower limbs (LL), contribute to the increase in heart rate ${ }^{(18,19)}$. There is also evidence that the active exercise of large muscle groups causes a decrease in blood pressure after exercise ${ }^{(20,21)}$. It is thus possible to justify 
Table 4. Comparison of time intervals according to the independent variables

\begin{tabular}{|c|c|c|c|c|c|c|}
\hline \multicolumn{7}{|c|}{ Heart Rate (beats/min) } \\
\hline & \multicolumn{2}{|c|}{ Rest $\rightarrow 5$ min activi. } & \multicolumn{2}{|c|}{ Rest $\rightarrow 10 \mathrm{~min}$ activi. } & \multicolumn{2}{|c|}{$10 \mathrm{~min}$ activi. $\rightarrow 10 \mathrm{~min}$ recov. } \\
\hline & Mean \pm SD & Value $p$ & Mean \pm SD & Value $p$ & Mean \pm SD & Value $p$ \\
\hline \multicolumn{7}{|l|}{ Gender } \\
\hline Male & $7.6 \pm 6.0$ & 0.209 & $9.3 \pm 6.2$ & 0.412 & $-7.7 \pm 2.0$ & 0.759 \\
\hline Female & $19.8 \pm 7.3$ & & $17.6 \pm 7.7$ & & $-8.6 \pm 2.3$ & \\
\hline \multicolumn{7}{|l|}{ Age } \\
\hline$<65$ years & $5.6 \pm 6.0$ & 0.097 & $6.4 \pm 6.2$ & 0.149 & $-8.8 \pm 2.0$ & 0.566 \\
\hline$\geq 65$ years & $21.3 \pm 6.7$ & & $20.6 \pm 7.1$ & & $-7.1 \pm 2.2$ & \\
\hline \multicolumn{7}{|c|}{ Length of hospital stay } \\
\hline$\leq 14$ days & $8.4 \pm 5.5$ & 0.204 & $7.8 \pm 5.5$ & 0.135 & $-9.5 \pm 1.7$ & 0.148 \\
\hline$>14$ days & $21.5 \pm 8.3$ & & $23.5 \pm 8.4$ & & $-4.8 \pm 2.5$ & \\
\hline \multicolumn{7}{|c|}{ Type of hospitalization } \\
\hline Clinical & $16.4 \pm 7.1$ & 0.471 & $16.6 \pm 7.3$ & 0.480 & $-7.8 \pm 2.2$ & 0.868 \\
\hline Surgical & $9.3 \pm 6.2$ & & $9.5 \pm 6.4$ & & $-8.3 \pm 2.0$ & \\
\hline
\end{tabular}

\begin{tabular}{|c|c|c|c|c|c|c|}
\hline \multicolumn{7}{|c|}{ Mean Arterial Pressure $(\mathrm{mmHg})$} \\
\hline & \multicolumn{2}{|c|}{ Rest $\rightarrow 5$ min activi. } & \multicolumn{2}{|c|}{ Rest $\rightarrow 10 \mathrm{~min}$ activi. } & \multicolumn{2}{|c|}{$10 \mathrm{~min}$ activi. $\rightarrow 10 \mathrm{~min}$ recov. } \\
\hline & Mean \pm SD & Value $p$ & Mean \pm SD & Value $p$ & Mean \pm SD & Value $p$ \\
\hline \multicolumn{7}{|l|}{ Gender } \\
\hline Male & $1.1 \pm 7.1$ & 0.276 & $-3.5 \pm 6.7$ & 0.345 & $-7.3 \pm 3.0$ & 0.871 \\
\hline Female & $13.8 \pm 8.8$ & & $6.8 \pm 8.4$ & & $-6.5 \pm 3.8$ & \\
\hline \multicolumn{7}{|l|}{ Age } \\
\hline$<65$ years & $3.1 \pm 7.5$ & 0.556 & $-4.2 \pm 7.0$ & 0.315 & $-4.0 \pm 3.0$ & 0.139 \\
\hline$\geq 65$ years & $10.0 \pm 8.6$ & & $6.7 \pm 8.0$ & & $-11.0 \pm 3.4$ & \\
\hline \multicolumn{7}{|c|}{ Length of hospital stay } \\
\hline$\leq 14$ days & $3.4 \pm 6.7$ & 0.480 & $-3.5 \pm 2.8$ & 0.258 & $-6.8 \pm 2.8$ & 0.885 \\
\hline$>14$ days & $12.2 \pm 10.2$ & & $9.7 \pm 9.4$ & & $-7.5 \pm 4.3$ & \\
\hline \multicolumn{7}{|c|}{ Type of hospitalization } \\
\hline Clinical & $7.4 \pm 8.6$ & 0.848 & $0.7 \pm 8.1$ & 0.977 & $-7.4 \pm 3.6$ & 0.987 \\
\hline Surgical & $5.1 \pm 7.6$ & & $0.3 \pm 7.1$ & & $-6.7 \pm 3.1$ & \\
\hline
\end{tabular}

\begin{tabular}{|c|c|c|c|c|c|c|}
\hline \multicolumn{7}{|c|}{ Systolic Blood Pressure (mmHg) } \\
\hline & \multicolumn{2}{|c|}{ Rest $\rightarrow 5$ min activi. } & \multicolumn{2}{|c|}{ Rest $\rightarrow 10$ min activi. } & \multicolumn{2}{|c|}{$10 \mathrm{~min}$ activi. $\rightarrow 10 \mathrm{~min}$ recov. } \\
\hline & Mean \pm SD & Value $p$ & Mean \pm SD & Value $p$ & Mean \pm SD & Value $p$ \\
\hline \multicolumn{7}{|l|}{ Gender } \\
\hline Male & $10.0 \pm 5.7$ & 0.924 & $6.1 \pm 4.7$ & 0.748 & $-14.0 \pm 4.4$ & 0.215 \\
\hline Female & $9.1 \pm 7.2$ & & $3.6 \pm 6.0$ & & $-4.8 \pm 5.5$ & \\
\hline \multicolumn{7}{|l|}{ Age } \\
\hline$<65$ years & $11.6 \pm 6.0$ & 0.622 & $9.0 \pm 4.7$ & 0.248 & $-9.1 \pm 4.7$ & 0.697 \\
\hline$\geq 65$ years & $7.1 \pm 6.7$ & & $0.3 \pm 5.4$ & & $-12.0 \pm 5.4$ & \\
\hline \multicolumn{7}{|c|}{ Length of hospital stay } \\
\hline$\leq 14$ days & $8.3 \pm 5.3$ & 0.671 & $2.7 \pm 4.3$ & 0.325 & $-9.3 \pm 4.2$ & 0.671 \\
\hline$>14$ days & $12.5 \pm 8.1$ & & $10.7 \pm 6.5$ & & $-12.7 \pm 6.4$ & \\
\hline \multicolumn{7}{|c|}{ Type of hospitalization } \\
\hline Clinical & $15.0 \pm 6.6$ & 0.297 & $6.1 \pm 5.6$ & 0.829 & $-7.1 \pm 5.3$ & 0.422 \\
\hline Surgical & $5.5 \pm 5.8$ & & $4.4 \pm 5.0$ & & $-13.0 \pm 4.6$ & \\
\hline
\end{tabular}

min.: Minutes; mmHg: Millimeters of Mercury; activi.: Activity; recov.: Recovery; SD.: Standard Deviation. Results expressed as the means \pm standard deviation, adopting the significance level of $p<0.05$. 
the increase in heart rate during exercise and the decrease in MAP and SBP in post-exercise, in our study, since the pedaling cycle ergometer involves the activation of large muscle groups such as quadriceps, hamstrings and triceps sural.

A study of therapeutic intervention was performed in an ICU, with elderly patients in postoperative coronary artery bypass surgery. The subjects were divided into 3 groups: group A performed cycle ergometer, B performed physical therapy without the use ergometer and the $C$ group did not perform any motor activity, and used non-invasive ventilation (NIV). Group A it performed cycle ergometer with intensity of 30 revolutions per minute, in five series three minutes, with 1 minute break between sets. An increase in HR and a decrease in SBP were noted at posttest compared to pre-test ${ }^{(22)}$. Similar response was found in the present study where the HR increase and decrease in SBP was observed when comparing the 10 minutes after activity with rest.

It was observed in this study that the PAM values and PAS after 10 minutes of recovery were lower when compared to baseline. This fact can be explained by post-exercise hypotension $(\mathrm{PEH})$, but there is no consensus on how the exercise should be performed to obtain a good response from it. The physiological mechanism for HPE is not yet well established, but it is possible to infer that its occurrence is related to a number of factors that influence the peripheral vascular resistance and cardiac output. There are studies that show that aerobic exercise lasting 20-60 minutes have higher

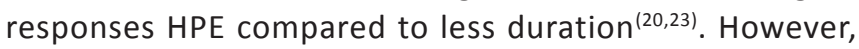
MacDonald et al (2000) conducted a study with sedentary individuals of both genders, who did a cycle ergometer for 10 minutes; the authors demonstrated a reduction in blood pressure at the end of the activity, thus corroborating our data, where there was a decrease in MAP and SBP compared to baseline $(-7.14 \%$ and $-7.90 \%$, respectively).

In our study, the clinical patients presented higher HR variations, PAM and PAS and are more likely to present an increase in changes of hemodynamic signals when subjected to an aerobic exercise compared to surgical patients. It is suggested that this occurs due to the fact that the reason for ICU admission turns to be the worsening of clinical status. Because they are more vulnerable to have complications that require mechanical ventilation, use of beta-blockers, antibiotics, and corticosteroids, which cause more hospitalization and more prolonged rest, causing a cascade of systemic changes.

With the emergence of some exercise programs for cancer patients, and the relative lack of guidelines for health professionals for dealing with this particular population, it became necessary to form guidelines to facilitate compliance with this group before, during and after their treatment program. There are no guidelines for the management of cancer patients during their hospital stay in intensive care units yet. However, the existing indications for cancer patients are similar to those suggested for healthy individuals as recommended by the American College of Sports Medicine $(A C S M)^{(25)}$. Through this study we observed that our results are similar to those found in other studies with hypertensive, cardiac and surgical non-cancer, patients showing that physical exercise appears to be safe and feasible.

Some limitations were observed in this study: 1 . there was no standardization of cadence performed by the patient, which may underestimate the results obtained; 2 . we did not check the patient's activity profile, possibly the most active preoperatively are those who have shown the best results.

\section{CONCLUSION}

During and after the activity, it was observed that the female subjects, aged greater than 65 years, with a length of stay greater than 14 days and who were submitted to clinical treatment showed greater variation in heart rate, mean arterial blood pressure and systolic blood pressure. No subject experienced signs of intolerance while performing the exercise.

Despite the small number of study participants, we observed in this group that the hemodynamic changes presented are similar to other groups of non-cancer patients. Thus, it appears that the cycle ergometer is a viable resource in the management of critical cancer patients, and it can be used for physical acitivities in the ICU program.

\section{AUTHORS' CONTRIBUTIONS}

Wylisson Marcelo Almeida Lins: Conception and design; Acquisition of data; Interpretation of data; Writing the manuscript. Ana Cristina Machado Leão: Conception and design; Interpretation of data; Writing the manuscript; Critical review; Final approval. Anke Bergmann: Conception and design; Interpretation of data; Statistical analysis; Writing the manuscript; Critical review.

\section{CONFLICTS OF INTEREST}

The author(s) declare that they have no competing interests.

\section{REFERENCES}

1. Soares M, Carvalho M, Salluh J, Ferreira C, Luiz R, Rocco J et al. Effect of age on survival of critically ill patients with cancer. Crit Care Med. 2006; PAP.

2. Courneya K. Exercise in Cancer Survivors: An Overview of Research. Med Sci Sports Exerc. 2003; 35(11):1846-1852.

3. Desai S, Law T, Needham D. Long-term complications of critical care. Crit Care Med. 2011; 39(2): 371-379.

4. Perme C, Chandrashekar R. Early Mobility and Walking Program for Patients in Intensive Care Units: Creating a Standard of Care. Am J Crit Care. 2009;18(3):212-221.

5. Topp R, Ditmyer M, King K, Doherty K, Hornyak J. The Effect of Bed Rest and Potential of Prehabilitation on Patients in the Intensive Care Unit. AACN Adv Crit Care. 2002; 13(2):263-276.

6. Stiller K. Safety Issues That Should Be Considered When Mobilizing Critically III Patients. Crit Care Clin. 2007; 23(1):35-53.

7. Morris P, Goad A, Thompson C, Taylor K, Harry B, Passmore L et al. Early intensive care unit mobility therapy in the treatment of acute respiratory failure. Crit Care Med. 2008; 36(8): 2238-2243.

8. Feliciano V, Albuquerque C, Andrade F, Dantas C, Lopez A, Ramos F et al. A influência da mobilização precoce no tempo de internamento na Unidade de Terapia Intensiva. ASSOBRAFIR Ciência. 2012; 03(02): 31-42. 
9. Burtin C, Clerckx B, Robbeets C, Ferdinande P, Langer D, Troosters T et al. Early exercise in critically ill patients enhances short-term functional recovery. Crit Care Med. 2009; 37(9): 2499-2505.

10. Pires-Neto R, Pereira A, Parente C, Sant'Anna G, Daguer Esposito D, Kimura A et al. Caracterização do uso do cicloergômetro para auxiliar no atendimento fisioterapêutico em pacientes críticos. Rev Bras Ter Intensiva. 2013; 25(1): 39-43.

11. Teasdale G, Jennett B. Assessment of coma and impaired consciousness. The Lancet. 1974;304(7872):81-84.

12. Bohannon, R. and Smith, M. (1987). "Interrater reliability of a modified Ashworth scale of muscle spasticity." Physical Therapy 67(2): 206.

13. Wilmore J, Costill D, Kenney L. Fisiologia do esporte e do exercício. 5a ed. Barueri: Manole; 2013.

14. Guyton A, Hall J. Tratado de fisiologia médica. Madrid: Elsevier; 2011.

15. Stiller K, Phillips A. Safety aspects of mobilising acutely ill inpatients. Physiother Theory Pract. 2003; 19(4):239-257.

16. Weissman C. Effect of routine intensive care interactions on metabolic rate. CHEST J. 1984; 86(6):815.

17. Stiller K, Phillips A, Lambert P. The safety of mobilisation and its effect on haemodynamic and respiratory status of intensive care patients. Physiother Theory Pract. 2004; 20(3):175-185.
18. Farinatti P, Soares P, Monteiro W, Duarte A, Castro L. Cardiovascular responses to passive static flexibility exercises are influenced by the stretched muscle mass and the Valsalva maneuver. Clinics. 2011; 66(3):459-464.

19. Freitas E, Bersi R, Kuromoto M, Slembarski S, Sato A, Carvalho M. Efeitos da mobilização passiva nas respostas hemodinâmicas agudas em pacientes sob ventilação mecânica. Rev Bras Ter Intensiva. 2012; 24(01): 72-78.

20. MacDonald J, MacDougall J, Hogben C. The effects of exercise duration on post-exercise hypotension. J Hum Hypertens. 2000; 14(2):125-129.

21. Jones H, Pritchard C, George K, Edwards B, Atkinson G. The acute postexercise response of blood pressure varies with time of day. Eur J Appl Physiol. 2008; 104(3):481-489.

22. Almeida K, Novo A, Carneiro S, Araújo L. Análise das Variáveis Hemodinâmicas em Idosos Revascularizados após Mobilização Precoce no Leito. Rev Bras Cardiol. 2014; 27(03): 165-171.

23. Moraes M, Bacurau R, Ramalho J, Reis F, Casarini D, Chagas J et al. Increase in kinins on post-exercise hypotension in normotensive and hypertensive volunteers. Biol Chem. 2007; 388(5).

24. MacDonald J, MacDougall J, Hogben C. The effects of exercising muscle mass on post exercise hypotension. J Hum Hypertens. 2000; 14(5):317320.

25. Schmitz K, Courneya K, Matthews C, Demark-Wahnefried W, Galvão D, Pinto B et al. American College of Sports Medicine Roundtable on Exercise Guidelines for Cancer Survivors. Med Sci Sports Exerc. 2010; 42(7): 14091426. 